Ciência e Natura, v. 37 Part 2 2015, p. 399-405

ISSN impressa: 0100-8307 ISSN on-line: 2179-460X

\title{
ciênciaenatura
}

\section{Multicast Data Replication Approach for Improving Fault Tolerance in Mobile Ad hoc Networks}

\author{
Sogand Sahabi Moghaddam ${ }^{1}$, Abbas Karimi 1,* \\ ${ }^{1}$ Department of Computer Engineering, Faculty of Engineering, Arak Branch, Islamic \\ Azad University, Arak, Iran \\ *Akarimi@iau-arak.ac.ir
}

\begin{abstract}
Abstract Multicast data replication provides a possible solution for improving data accessibility in highly dynamic and fault prone mobile ad hoc environments. Our novel multicast data replication approach operates in a self-organizing manner where the network nodes that has unit host detector construct a connected dominating set (CDS) based on the topology graph by collecting information from neighboring nodes using multicast if gathered data from neighbors have two non-adjacent neighbors then use that virtual backbone for efficient data replication, data search and routing. In this study, we compare our proposed approach with SCALAR and evaluate it in average hop counts and successful delivery ratio with different node numbers and speeds.

It is shown that the average hop counts increased but with falling rate and 20 percent successful delivery ratio is achieved, so it is demonstrated that PM act with respect to fault tolerance improvement, power consumption and load balancing is occurred.

Keywords: mobile ad hoc network1, data replications2, fault tolerance3, multicast4, CDS5.
\end{abstract}




\section{Introduction}

$\mathrm{M}$

obile ad hoc network is a type of wireless ad hoc networks. It is a collection of mobile nodes which can dynamically exchange information between nodes without any centralized infrastructure. These networks can be well suited for an environment where either the infrastructure is lost or where deploy an infrastructure is not very cost effective [2]. However in this case, the nodes are limited to send and receive information but do not route anything across the network. It can turn the dream of getting connected "anywhere and at any time" into reality. Typical application examples include a disaster recovery or a military operation.

\subsection{Need of Data Replication}

In order to prevent data loss and increase public data access in case of unpredictable situation due to rising mobile node, the public data replication used. As well as data replication, performance improved by reducing the number of nodes between the sender and the destination node [4]. As far as possible, the goals of these networks are based on determining the necessary data and send it to the requesting node in which each node transmits the data itself by using multicast that is a method to avoid traffic and keep data in order not to lose it.

Mobile hosts often get disconnected from the network due to various factors like power failure or their mobility. Servers which hold the data cannot provide services if they are disconnected from other mobile hosts [7]. Thus, ideally, the replication method should be able to determine when a particular mobile host would be disconnected and, accordingly, replicate its data items in a different server to improve data accessibility.

\subsection{Fault Tolerance}

The concept of fault tolerance in network, creates strategies to prevent failure in the network. Due to node mobility the topology of the network can change frequently, nodes can move away from the transmission range. Every node in the network can communicate with the other nodes be located in the transmission range. For communication with the nodes, that node is not lie within the particular range, the nodes uses the middle node to pass the information to the hop. In case, the node is not in the transmission range node failure will occur. During this communication, the power of each node's battery is used for the route selection, discovery and repair the failures in the link of network. So, transmission power and energy is also important in this network.

As a result, there may be a chance of node failure or link failure and node have to tolerate fault to transfer the packets from source to destination easily. The performance of the routing will be decreased because of these failures [16].

The remainder of the paper is organized as follows. In section 2, we introduce some related works. In section 3, we describe our assumed structure. In section 4 , we propose a multicast data replication approach; then in section 5 , we show the results of performance analysis. In section 6, we summarize this paper.

\section{Related Work}

Data replication is a popular research topic in Mobile ad hoc networks, hence the well-known one was introduced by Hara and Madryda et al. protocols such as SAF, DAFN and DCG suggested. In conventional distributed systems, replication will not only increase data access but also helps distribute symmetric load and fault tolerance, but proposed protocols for data replication in these networks with increasing network size usually associated with problems such as causing errors in the system [1] to [4].

Atstan et al in [3] SCALAR performance have compared with other data replication methods such as SAF and DAFN. SCALAR protocol is based on the P2P network where each node acts as both the recipient and the sender. This method uses data replication and data control simultaneously. Lack of versatility with 
increasing the volume of network is disadvantage of this protocol.

In [5] a way to solve the problem of mobile data networks access based on data replication is proposed. At first, three methods of allocation replica suggested and assuming that the data are not updated. In all three proposed method of mobile data access, servers and network connection status is investigated. The same method for the conventional update of the data was developed.

The main cause of errors in mobile ad hoc networks is mobility nodes and introduced energy constraints. In this way, real-time mobile ad hoc data replication for databases called DREAM suggested [6] and [7].

In [9] a model for large-scale data replication in MANETs suggested. DHTR clusters all mobile nodes that do not overlap. To speed up referral uses the hash table, DHTR is distributed.

Performance and accuracy of the results in mobile networks depends on the number and location generated replica. It is suggested that the nodes should share the heavy loads in order to strike a balance, then according to demand and the network topology, transferring and making a replica was performed [10] and [11].

In addition to addressing, consistency and availability of data in conventional databases, the conditions of data replication technique in these networks such as energy consumption [8] and dynamism of servers, mobility and energy consumption of clients, real-time applications and these networks' configuration were considered. We found that energy consumption requires real-time application and network configuration, there are three important issues in the design of the case in mentioned networks. These issues are considered normal networks but in MANETs, it is related to providers. These networks allow servers and clients to move, so disconnection and reconfiguration occurs most frequently in these networks.

In this paper, multicast data replication model for improving fault tolerance was proposed and results compare with SCALAR protocol. Results show PM act better than SCALAR protocol and it leads to improve fault tolerance, increase data access, achieve load balancing [10] and power consumption.

\section{Assumptions}

In this section, a comparison between SCALAR protocol used to intend medium to large-scale mobile ad hoc network. Each node in this protocol has unit host detector [4]. The set of nodes is defined with $\mathrm{M}=\{\mathrm{M} 1, \mathrm{M} 2, \ldots$, $\mathrm{MN}$, where $\mathrm{N}$ represents the total number of nodes. Initially, each node $\mathrm{Mi}$ is the owner of data item di, where data set are shown as $\mathrm{D}=$ $\{\mathrm{d} 1, \mathrm{~d} 2, \ldots, \mathrm{dN}\}$. Each node, $\mathrm{Mi}$, can save a copy of the data items, defined as D, limited with its memory. Each node, $\mathrm{Mi}$, is aware of existing data set in $\mathrm{D}$ and can request any data item $\mathrm{dj}$ at any time [13].

\subsection{Virtual Backbone Construction Using Connecting Dominating Set (CDS)}

CDS in mobile ad hoc network is used for the construction of a virtual backbone. It is a graph shown in Figure 1 that has a following properties:

Each node requires only single hop information about this network. Resulting set of nodes forms a dominating set; and every one of them is directly connected to at least one other dominator node. All other nodes, which do not exist in the CDS, are directly connected to at least one node in the resulting set.

The process starts with a neighbor list exchange among nodes. Then each node collects information from neighboring nodes using multicast, after gathering data from neighbors, If and only if they have two non-adjacent neighbors, they introduce themselves as a dominating node (a member of the CDS). Then the pruning phase was started. In this phase some node removes itself from the CDS. CDS recalculation performed by periodically invoking the mentioned construction and there is no guarantee that nodes determined as dominator form a CDS at any time. As a result, this process message complexity is $\Theta(n)$, where $\mathrm{n}$ is the number of nodes in the network [13].

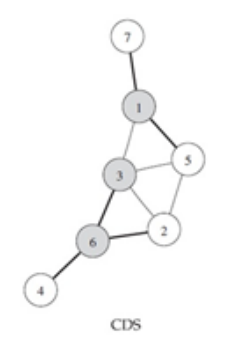

Figure 1: CDS graph 


\section{Multicast Data Replication Approach}

In this section, we explain a multicast data replication approach with some new assumption regarding SCALAR [3].

In SCALAR when a node that is not a member in backbone, requests a data. At first the request must be sent to the backbone then search should be performed. In the proposed approach, a node that is not a member of the backbone, just using broadcast method for sending its request to neighboring nodes in order to perform searching process in those nodes who are not members of the backbone. If the neighboring nodes (node which is not a member of the backbone) have desired data, sends it to the requesting node otherwise, it ignores this request then request forwarding is avoided (nodes that are not members of the backbone do not allow to forward data).

The process of data searching and forwarding must perform via the backbone members and it is done by broadcasting request. When one of the members receives this request, continues search with broadcasting, if this request has not received before, otherwise, rejects it [12] and [15].

Search process continues until finding desired data. Then data requested back with the same path into node who requested data. During the return path, each node decides whether or not to keep a copy of the data. This decision is based on historic requests and will be compared with the current data value. The proposed approach assumes that each node has limited memory capacity to save data (assuming that each node has the memory to store up to 5 items) [13] and [15].

\section{Performance Evaluation}

In this section, we show simulation results to investigate the performance of proposed approach by using the MATLAB simulator.

\subsection{Simulation Design}

Our network model contains mobile nodes over a terrain of size $1500^{*} 1500$ square meters. As it has been shown in figure 2 (in each part a and $b$, right part shows the mobile node and left part shows backbone graph), over defined time as t0 and $t 1$, node position changed and this change can also affect the backbone graph.
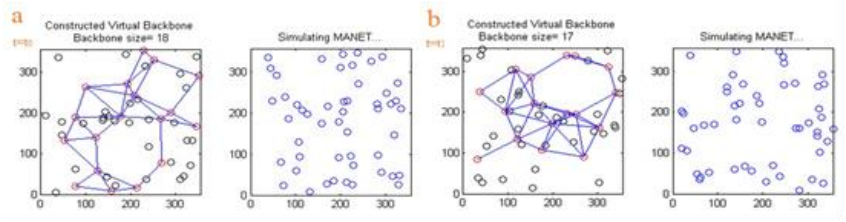

Figure 2: Network structure during $\mathrm{t} 0$ and $\mathrm{t} 1$

We use SCALAR (scalable data lookup and replication protocol) by Atstan et al. [13] in order to compare to our approach and measure hop count and successful delivery ratio by increasing nodes and speeds during simulation and total simulation time lasts 900 seconds, in which each scenario lasts 5000 milliseconds.

The performance metrics of interest to us are: (1) average number of hops - the average path length used to transmit the packet from source to destination. (2) successful delivery ratio - the successful ratio of data packets received by the destinations to those generated by the sources.

\subsection{Numerical Results}

We emphasize the effects of two important factors in large scale ad hoc networks, namely the number of mobile nodes and their speeds that can greatly affect the performance of our approach. We use selected number of nodes between 40 and 500 shown in Table 1 that move at a specific speed uniformly selected between 1 and 7 meters /second shown in Table 2.

Table 1. node numbers matrix

\begin{tabular}{cccc}
\hline $\mathrm{N}_{1}$ & $\mathrm{~N}_{2}$ & $\mathrm{~N}_{3}$ & $\mathrm{~N}_{4}$ \\
\hline 50 & 100 & 200 & 400 \\
\hline
\end{tabular}


Table 2. node speeds matrix

\begin{tabular}{ccccc}
\hline $\mathrm{S}_{1}(\mathrm{~m} / \mathrm{s})$ & $\mathrm{S}_{2}$ & $\mathrm{~S}_{3}$ & $\mathrm{~S}_{4}$ & $\mathrm{~S}_{5}$ \\
\hline 1 & 2.5 & 4 & 5.5 & 7 \\
\hline
\end{tabular}

During the time, in the set of experiments we investigate the impact of the number of nodes and speeds. Simulation time for each scenario lasts 5000 milliseconds.

Figure 3 shows successful delivery ratio with specified number of nodes that is mentioned in Table1. In initial time this ratio increases fast then after passing time, because of congestion this ratio decreases as the number of nodes increase.

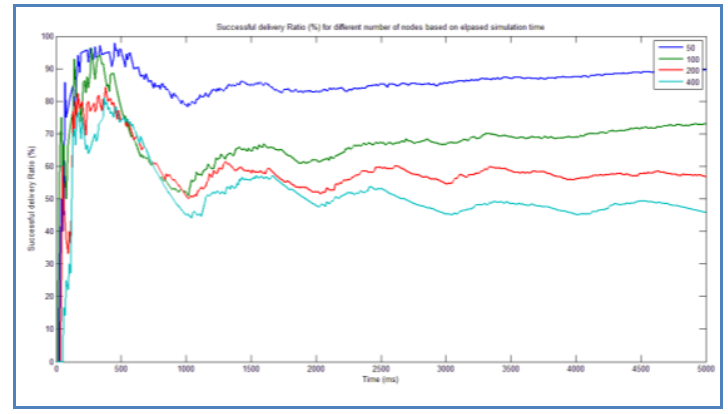

Figure 3: Successful delivery ratio by changing node numbers

Figure 4 shows successful delivery ratio with specified speed of each nodes that is mentioned in Table2. In initial time this ratio increases fast then after passing time, because of node scattering this ratio decreases as the node speeds increase. So, network stability is approximately achieved by passing time.

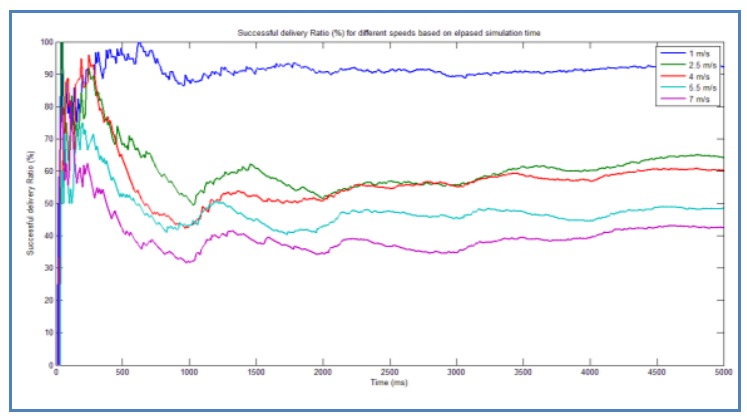

Figure 4: Successful delivery ratio by changing node speeds

In the following, we also examine the performance of proposed approach by comparing it with SCALAR.
Figure 5 shows the average number of hop counts used to transmit the data packet. In SCALAR with an increase in the number of nodes, number of hop counts almost increases linearly. On the other hand, in the proposed method with an increase in the number of mobile nodes, the average hop counts increased but with falling rate. It means that the performance of proposed approach is suitable in large scale network.

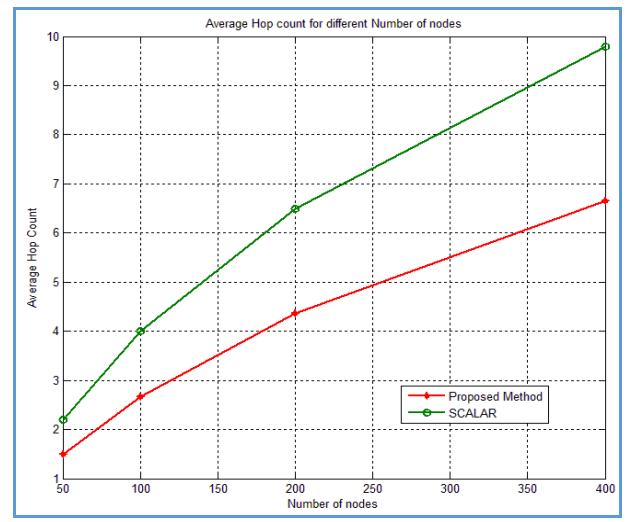

Figure 5: Proposed method vs SCALAR in average hop count

Figure 6 shows successful delivery ratio vs increasing in the network space. Both graphs show a similar behavior, but the proposed approach by almost all successful delivery ratio is $20 \%$ better than SCALAR.

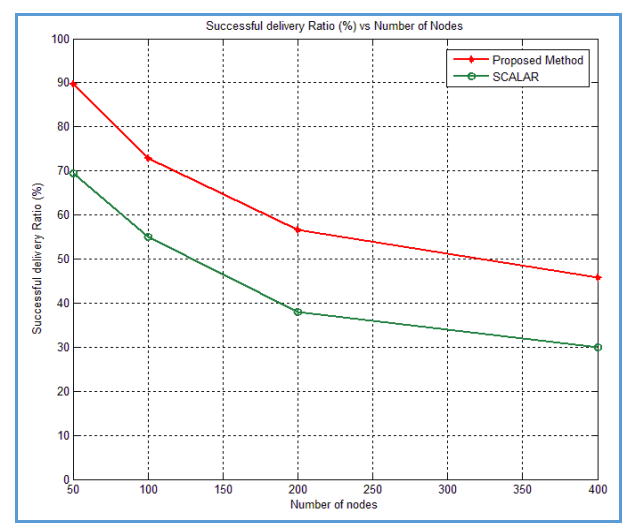

Figure 6: Proposed method vs SCALAR in successful delivery ratio

\section{Conclusion}

In recent year, with the development of computer technology, the necessity of wireless communications such as mobile ad hoc networks 
has been considered [1]. In these self-organizing networks, fault tolerant data access is a main point to facilitate communications hence, we proposed multicast data replication approach for improving fault tolerance in mobile ad hoc networks. It is a fault tolerant solution for handling such errors generated by different phases of replication and multicasting process so that it can be easily adapted to large scale network scenarios. On the other hand, it is as successful as other data replication methods in constructing virtual backbone and search and forward data packets in which each node in virtual backbone has unit host detector and collects information from neighboring nodes using multicast then best possible options can be selected as a member of the CDS, if gathered data from neighbors have two non-adjacent neighbors so it is a best-effort solution to construct CDS in mobile ad hoc networks.

We compared the proposed approach with SCALAR in different number of nodes between 40 and 500 and their movement speeds uniformly selected between 1 and 7 meters /second then evaluate it in number of hop counts and successful delivery ratio of data packets.

Simulation results show that our proposed approach performs better than SCALAR with fault tolerance improvement in terms of data accessibility by falling average rate of hop counts and good performance by achieving 20\% successful delivery ratio, furthermore it can perform quite well in transmission power and energy consumption in high node density.

\section{References}

[1] Misra, S., Woungang, I., Misra, S.C. (2009). Guide to Wireless Ad Hoc Networks. London: Springer-Verlag.

[2] Tavli, B., Heinzelman, W. (2006). Mobile Ad Hoc Networks: Energy-Efficient Real-Time Data Communications. Dordrecht: Springer.

[3] Atsan, E., Altinbuken, D., Ozkasap, O. (2009). SCALAR data replication performance in mobile ad hoc applications. IEEE 24th International Symposium on Computer and Information Sciences (ISCIS), Guzelyurt, p. $369-374$.

[4] Hara, T., Madria, S.K. (2006). Data Replication for Improving Data Accessibility in Ad Hoc
Networks. IEEE Transactions on Mobile Computing. vol. 5, no. 11, p. 1515 - 1532.

[5] Mondal, A., Madria, S.K., Kitsuregawa, M. (2006). CADRE: A Collaborative replica allocation and deallocation approach for Mobile-P2P networks. IEEE 10th International Database Engineering and Applications Symposium (IDEAS'06), Delhi, p. 21-28.

[6] Pabmanabhan, P., Gruenwald, L. (2006). DREAM: A Data Replication Technique for Real-Time Mobile Ad-hoc Network Databases. IEEE Proceedings of the 22nd International Conference on Data Engineering (ICDE'06), Washington DC.

[7] Derhab, A., Badache, N. (2009). Data replication protocols for mobile ad-hoc networks: a survey and taxonomy. IEEE Communications Surveys \& Tutorials, vol. 11, no. 2, p. 33-51.

[8] Yu, H., Martin, P., Hassanein, H. (2005). Cluster-based replication for large-scale mobile ad-hoc networks. IEEE International Conference on Wireless Networks, Communications and Mobile Computing, vol. 1, p. 552 - 557.

[9] La, C., Michiardi, P., Casetti, C., Chiasserini, C., Fiore, M. (2010). A Lightweight Distributed Solution to Content Replication in Mobile Networks. IEEE Wireless Communications and Networking Conference (WCNC), Sydney, p. 1-6.

[10]Torbey, Z., Bennani, N., Brunie, L., Coquil, D. (2011). Performance Evaluation for CReaM: User-Centric Replication Model. IEEE 11th Annual International Conference on New Technologies of Distributed Systems (NOTERE), Paris, p. 1-8.

[11]Sasson, Y., Cavin, D., Schiper, A. (2003). Probabilistic broadcast for flooding in wireless mobile ad hoc networks. IEEE Wireless Communications and Networking Conference (WCNC). New Orleans, vol. 2, p. 1124 - 1130.

[12]Hara, T., Madria, S.K. (2009). Consistency management strategies for data replication in mobile ad hoc networks. IEEE Transactions on Mobile Computing. vol. 8, no. 7, p. 950 967. 
[13]Atsan, E., Ozkasap, O. (2013). SCALAR: Scalable data lookup and replication protocol for mobile ad hoc networks. Journal of Computer Networks, vol. 57, no. 17, p. 36543672.

[14]Shinohara, M., Hara, T., Nishio, S. (2009). A Data Transmission Method using Multicast in Mobile Ad Hoc Networks. IEEE Tenth International Conference on Mobile Data Management: Systems, Services and Middleware (MDM), Taipei, p. 232-237.

[15] Jia, L. (2011). A Mobile Ad-hoc Network Data Cache Invalidation Method. Journal of Procedia Engineering, vol. 23, p. 150-154.

[16] Moraes, R.E.N., Ribeiro, C.C., Duhamel, C. (2009). Optimal solutions for fault-tolerant topology control in wireless ad hoc networks. IEEE Transactions on Wireless Communications, vol. 8, no. 12, p. 5970-5981. 